\title{
Superior Mesenteric Artery Thrombosis in COVID-19 Pneumonia: an Underestimated Diagnosis-First Case Report in Asia
}

\author{
Sunaina Tejpal Karna ${ }^{1}$ (D) $\cdot$ Rajesh Panda $^{1} \cdot$ Ajeet Pratap Maurya ${ }^{2} \cdot$ Shashi Kumari $^{1}$
}

Received: 12 May 2020 / Accepted: 14 October 2020 / Published online: 19 October 2020

(C) Association of Surgeons of India 2020

\begin{abstract}
The COVID-19 disease caused by novel coronavirus was first reported in Wuhan, China, in December 2019 with 5\% patients having severe lung injury. Though this disease primarily presents as a lower respiratory tract infection, multiple digestive manifestations have been reported which are often overlooked. The present case report describes the unusual progression of COVID-19 disease from pneumonia to a procoagulant state leading to superior mesenteric artery thrombosis and subsequent gut ischemia necessitating emergency laparotomy. Coagulopathy in COVID-19 is due to an imbalance in the coagulation homeostasis with increase in prothrombin time, fibrinogen, and D-dimers. Early recognition of abdominal symptoms, diagnosis of pathology, and timely surgical intervention may definitely improve outcome. In the management of any patient with COVID-19 disease, we advocate a comprehensive integrated approach with early recognition of digestive symptoms and their timely intervention which should run parallel to the respiratory management.
\end{abstract}

Keywords Superior mesenteric artery (SMA) thrombosis $\cdot$ Mesenteric ischemia $\cdot$ COVID-19 pneumonia

\section{Case Discussion}

A 61-year-old diabetic, hypertensive lady presented with respiratory distress to our Institute, a dedicated COVID-19 center with pulse110 bpm, blood pressure $140 / 78 \mathrm{mmHg}$, and room air saturation $78 \%$. Oxygen therapy was started with face mask. RT-PCR of paired nasopharyngeal and oral swab was positive for COVID-19 infection. In ICU, her routine blood investigations were sent (Table 1) and chest X-ray showed pneumonia pattern in the peripheral and basal region (Fig. 1). Treatment was started with high-flow nasal oxygen (HFNO), cefoperazone-sulbactam, prophylactic enoxaparin, pantoprazole, vitamin $\mathrm{C}$, and zinc as per institutional protocol. She showed symptomatic improvement with $\mathrm{SpO}_{2}$ of $95 \%$.

On the fourth day, she developed diffuse abdominal pain with distention. Contrast CT scan abdomen showed thrombosis of distal superior mesenteric artery (SMA) with dilated

Sunaina Tejpal Karna

drtejpal@gmail.com

1 Department of Anesthesiology and Critical Care, All India Institute of Medical Sciences, Saket Nagar, Bhopal, India

2 Department of Surgery, All India Institute of Medical Sciences, Bhopal, India jejunoileal loops and normal enhancing bowel wall. Unfractionated heparin 5000 units intravenous, followed by 1000 units/h infusion, was administered with Ecosprin and clopidogrel. Symptomatic relief with bowel movements was noted with anticoagulation therapy.

On the tenth day, acute abdominal pain and distention were noted with feculent vomiting. Emergency exploratory laparotomy planned after correction of coagulopathy revealed gangrenous distal ileum $60 \mathrm{~cm}$ in length $30 \mathrm{~cm}$ proximal to ileocecal junction. Mesentery was thickened and inflamed, no bleeding from distal mesenteric vessels, but pulsation is present in proximal superior mesenteric artery (Fig. 2). Small perforation is present in gangrenous bowel, and rest of small and large bowel were healthy. Resection of gangrenous bowel and loop ileostomy is done. Postoperative course was complicated by septic shock and acute renal failure. Despite maximal supportive care, she succumbed to the illness $36 \mathrm{~h}$ after surgery.

\section{Discussion}

We report the first case of superior mesenteric artery thrombosis in a patient with COVID-19 pneumonia in Asia. After favorable evolution of disease with anticoagulant therapy, 
Table 1 Serial trend of blood investigations: (1) $\mathrm{Hb}$ (hemoglobin), (2) TPC (total platelet count), (3) TLC (total leucocyte count), (4) NLR (neutrophil to lymphocyte ratio), (5) CRP (Creactive protein), (6) activated partial thromboplastin time, and (7) prothrombin time/ international normalized ratio

\begin{tabular}{lllllll}
\hline Parameters & $28 / 4 / 20$ & $30 / 4 / 20$ & $3 / 5 / 20$ & $6 / 5 / 20$ & $8 / 5 / 20$ & $9 / 5 / 20$ \\
\hline $\mathrm{Hb}^{1}$ in gm/dl & 12.4 & 11.2 & 10.4 & 9.4 & 8.7 & 7.1 \\
$\mathrm{TPC}^{2}$ in microliter & 401,000 & 464,000 & 520,000 & 574,000 & 579,000 & 311,000 \\
$\mathrm{TLC}^{3}$ per cubic millimeter & 21,300 & 17,000 & 11,600 & 12,000 & 18,200 & 23,000 \\
$\mathrm{NLR}^{4}$ & 10.2 & 19.3 & 10.5 & 12.5 & 15 & 18.5 \\
Monocyte $^{4}$ & 10 & 8 & 9 & 5 & 4 & 5 \\
$\mathrm{CRP}^{5}$ in mg/dl & 343 & 421.6 & 437 & 304 & 132 & 282 \\
Triglycerides in mg/dl $_{\text {aPTT }}^{6}$ & 105 & 466 & 712 & 672 & 411 & 105 \\
$\mathrm{PT} / \mathrm{INR}^{7}$ & & & 53 & 51.8 & 30.8 & 32.9 \\
Lactate mmol/1 & & & $20 / 1.8$ & $22 / 2.1$ & $21.3 / 2$ & $19 / 1.7$ \\
$\mathrm{pH}$ & 1.1 & 1.4 & 1.5 & 1.9 & 1.6 & 8.4 \\
\end{tabular}

mesenteric ischemia is manifested by acute abdominal pain. Though surgical exploration and gangrenous bowel resection were successful, the patient succumbed to vasoplegic shock and multi-organ dysfunction syndrome.

Thrombosis in severe COVID-19 may be due to inflammation; endothelial injury by viral affinity for ACE2 receptors in respiratory tract, heart, GI tract, and distal vasculature; activation of tissue factor pathway; excessive thrombin generation; increased fibrin formation; and polymerization with fibrinolysis shutdown [1]. Hypoxia in severe COVID-19 may stimulate thrombosis by increasing blood viscosity and a hypoxiainducible transcription factor-dependent signaling pathway [2]. The typical finding in COVID-19 coagulopathy is raised prothrombin time, fibrinogen, and D-dimers with a modest

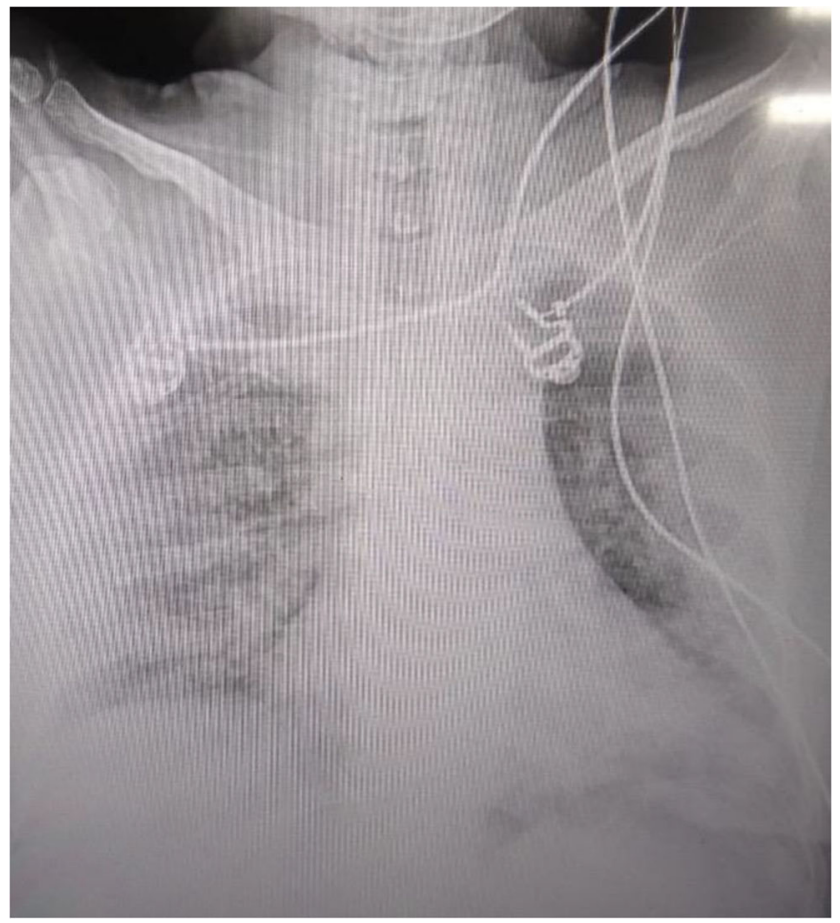

Fig. 1 Chest X-ray on day of admission X-ray decrease in platelet count with near normal activated partial thromboplastin time [1]. We observed an increase in prothrombin time with platelet count. The thrombocytosis may be reactive with the increase in acute phase reactants like CRP (Table 1) due to inflammation, increased thrombopoietin, and vasculitis [3].

Till now, only five cases of SMA thrombosis in COVID-19 positive patients are reported in the world [4-6]. Of these, one patient with multiple comorbidities, managed conservatively, did not survive. In the 4 operated patients, only two (28-yearold woman, 52-year-old man) survived while one (56-yearold man) was still on ventilatory support. Surgical procedures performed were jejunal resection with laparostomy followed by double jejunostomy and abdominal wall closure 2 days later (28-year-old female), bowel resection and side-to-side stapled anastomosis (52-year-old man). Microthrombi and inflammatory mediators were postulated to cause mesenteric ischemia. Though anticoagulation did improve disease evolution, sudden abdominal pain was noted in both cases. CT scan was diagnostic in both cases.

Early treatment with heparin is recommended by most literature in hospitalized patients with COVID-19 [1, 4-6]. Heparin has anticoagulant, anti-inflammatory, endothelial protective role in COVID-19. It can impact the microcirculatory dysfunction and possibly decrease organ damage. However, the appropriate dose in COVID is still a matter of ongoing research [7]. It is prudent to monitor prothrombin time, platelet count, and D-dimer concentrations in severe COVID-19 infection.

Mesenteric ischemia should be treated with early fluid resuscitation, use of broad-spectrum antibiotics and intravenous unfractionated heparin, and early intervention. The first $12 \mathrm{~h}$ between onset of symptom to treatment are crucial to perform vascular surgery effectively without requiring intestinal resection for good outcome [8]. However, either patients with COVID-19 present late or treatment is focused on respiratory symptoms. Mucosal ischemia may induce massive viremia 
Fig. 2 CT cut section showing patent proximal superior mesenteric artery with occlusion of distal ileocolic branch
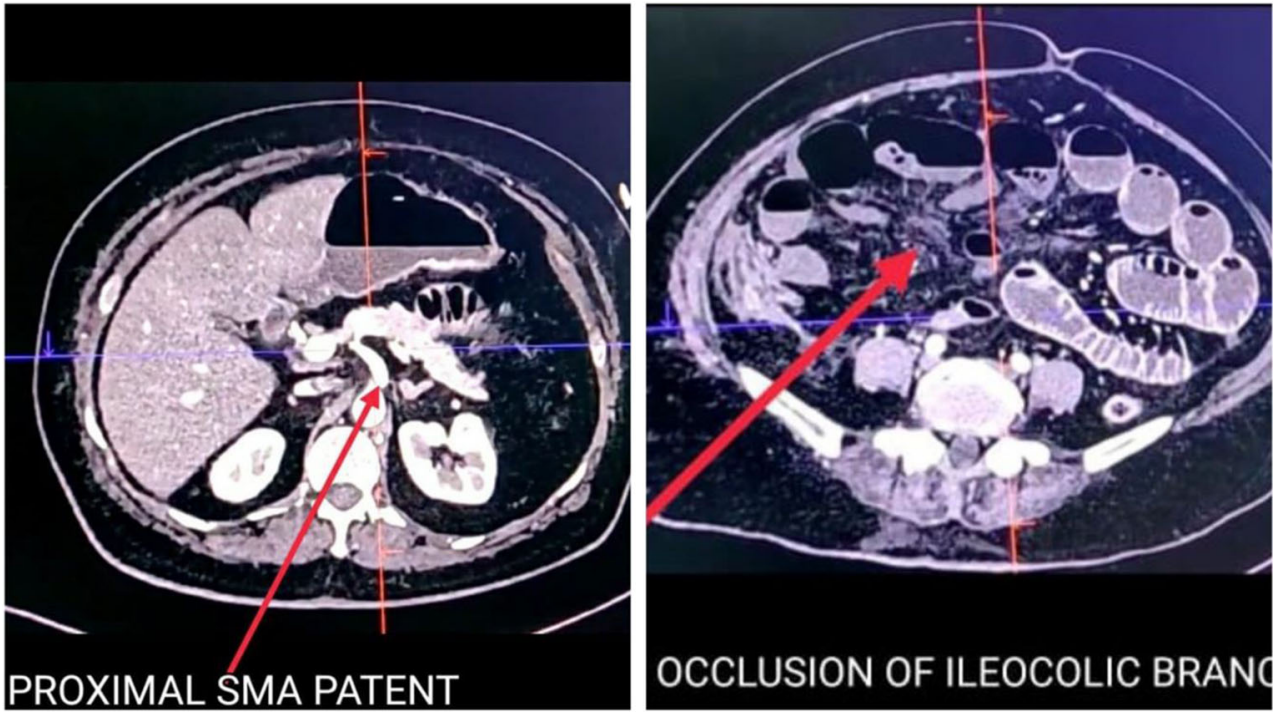

from bowel epithelium causing vasoplegic shock after surgery, as was noted in our case.

Patients with COVID-19 disease should be managed with a holistic approach with evaluation of digestive symptoms along with respiratory. In all patients with COVID-19 disease, routine anticoagulation with heparin with regular monitoring of coagulation tests may prevent thrombotic complications. Mesenteric ischemia should be suspected if the patient has abdominal distension or pain with increased inflammatory markers. Contrast-enhanced CT scan should be done for diagnosis and may be repeated to see the progression of disease. Early intervention is lifesaving. Emergency laparotomy may have a favorable outcome if done immediately after onset of abdominal pain and before onset of any new organ failure.

\section{Compliance with Ethical Standards}

Conflict of Interest The authors declare no conflict of interest.

\section{References}

1. Cao W, Li T (2020) COVID-19: towards understanding of pathogenesis. Cell Res 28:1-3
2. Gupta N, Zhao YY, Evans CE (2019) The stimulation of thrombosis by hypoxia. Thromb Res 181:77-83

3. Yin S, Huang M, Li D, Tang N (2020) Difference of coagulation features between severe pneumonia induced by SARS-CoV2 and non-SARS-CoV2. J Thromb Thrombolysis 3:1

4. Beccara L, Pacioni C, Ponton S, Francavilla S, Cuzzoli A (2020) Arterial mesenteric thrombosis as a complication of SARS-CoV-2 infection. Eur J Case Rep Intern Med 7(5). https://doi.org/10.12890/ 2020001690

5. de Barry O, Mekki A, Diffre C, Seror M, Hajjam ME, Carlier RY (2020) Arterial and venous abdominal thrombosis in a 79-year-old woman with COVID-19 pneumonia. Radiol Case Rep 15:1054 1057. https://doi.org/10.1016/j.radcr.2020.04.055

6. Ignat M, Philouze $\mathrm{G}$, Aussenac-Belle L, Faucher V, Collange O, Mutter D, Pessaux P (2020) Small bowel ischemia and SARSCoV-2 infection: an underdiagnosed distinct clinical entity. Surg J 168:14-16. https://doi.org/10.1016/j.surg.2020.04.035

7. Jagielski M, Piątkowski J, Jackowski M (2020) Challenges encountered during the treatment of acute mesenteric ischemia. Gastroenterol Res Pract. https://doi.org/10.1155/2020/5316849

8. Thachil J (2020) The versatile heparin in COVID-19. J Thromb Haemost 18(5):1020-1022

Publisher's Note Springer Nature remains neutral with regard to jurisdictional claims in published maps and institutional affiliations. 